\title{
VERIFICATION OF COMPATIBILITY OF ISOTROPIC CONSOLIDATION CHARACTERISTICS OF SOILS TO MULTIPLICATIVE DECOMPOSITION OF DEFORMATION GRADIENT
}

\author{
KOICHI HASHIGUCHI ${ }^{\mathrm{i})}$
}

\begin{abstract}
It is verified that the linear relation of logarithmic volumetric strain $v s$. logarithm of pressure derived from the linear relation of both logarithms of volume and pressure (Hashiguchi, 1974) for isotropic consolidation of soils is exactly compatible to the multiplicative decomposition of deformation gradient (Lee, 1969) which would be one of the fundamental requirements for constitutive equations describing the finite elastoplastic deformation. On the other hand, it is emphasized that the adoption of the linear relation of void ratio $v s$. logarithm of pressure is impertinent for the constitutive equation describing the finite deformation although it is most widely used for constitutive equations of soils.
\end{abstract}

Key words: constitutive equation, deformation gradient, elstoplasticity, isotropic consolidation, multiplicative decomposition, volumetric strain (IGC: D6/E2)

\section{INTRODUCTION}

Soils often undergo a large deformation in soil structures and thus it is of importance to formulate constitutive equations capable of describing the finite deformation of soils for practical problems in engineering. In addition, in contrast to metals a large volume change is induced in soils and thus it influences significantly on the elastic and the plastic deformation characteristics of soils. Therefore, the isotropic consolidation characteristics have to be formulated pertinently in constitutive equation of soils. Especially, it has to be formulated strictly for the description of finite deformation of soils. The linear relation of void ratio vs. logarithm of pressure leading to the linear relation of nominal volumetric strain vs. logarithm of pressure is best-known as the isotropic consolidation characteristics of soils. On the other hand, the linear relation of both logarithms of volume vs. pressure leading to the linear relation of logarithmic volumetric strain vs. logarithm of pressure was proposed by the author (Hashiguchi, 1974, 1985; Hashiguchi and Ueno, 1977; Hashiguchi et al., 2002). The author has noticed that the former has various impertinent properties in both physical and mathematical aspects and in contrast the latter is pertinent physically and mathematically and the impertinences in the former can be excluded by the latter (Hashiguchi, 1995). However, the former is adopted still now widely to constitutive equations of soils although constitutive equation capable of describing a finite deformation of soils cannot be formulated as far as it is used.

In this article it will be verified that the above-mentioned linear relation of logarithm of volume $v s$. logarithm of pressure is exactly compatible to the multiplicative decomposition of deformation gradient into the elastic and the plastic parts (Lee, 1969) which would have to be fulfilled in constitutive equations describing a finite elastoplastic deformation. This relation is described by the logarithm of volume itself and thus it leads exactly to the linear relations of logarithmic volumetric strain $v s$. logarithm of pressure and of the volumetric strain rate $v s$. rate of pressure in the quite simple forms. It is based on the isotropic consolidation characteristics but is indispensable in order to formulate constitutive equations of soils the hardening/softening of which proceeds mainly by the plastic volumetric strain.

The signs of stress (rate) and strain (rate) components are taken to be positive for tension, and the stress for soils is meant to be the effective stress, i.e., the stress excluding pore pressure from the total stress throughout this article.

\section{MUTIPLICATIVE DECOMPOSITION OF DEFORMATION GRADIENT}

Variation in characteristics of mechanical response of materials is caused by irreversible changes of their internal structures. Here, the changes of internal structures are macroscopically induced by the plastic deformation but it has to be free from the elastic deformation. There-

i) Professor, College of Technology, Department of Civil and Environmental Engineering, Daiichi University, Japan (khashi@bpes.kyushuu.ac.jp)

The manuscript for this paper was received for review on November 15, 2007; approved on May 28, 2008.

Written discussions on this paper should be submitted before March 1, 2009 to the Japanese Geotechnical Society, 4-38-2, Sengoku, Bunkyoku, Tokyo 112-0011, Japan. Upon request the closing date may be extended one month. 
fore, the rigorous decomposition of deformation into the elastic and the plastic deformations is required for the description of elastoplastic deformation. Here, strictly speaking, the elastic deformation has to be described by the purely elastic, i.e., hyper-elastic constitutive equation in which the elastic deformation returns to an initial state during a stress cycle and the work done during that cycle is zero. Microscopically, the elastic deformation is induced by the deformations of material particles themselves and the plastic deformation is induced by the mutual slips between material particles. The decomposition of deformation into the elastic and the plastic deformations is to be the basic concept of elastoplasticity.

On the premise of the definite decomposition of the deformation into the elastic and the plastic deformations as described above, the individual constitutive relations are formulated for these deformations in the elastoplasticity. Then, one has first to decompose the current deformation into the elastic and the plastic deformations explicitly. The elastic deformation is known from the current stress since it is related uniquely to the stress, and then the plastic deformation is known as that remainder. This is replaced by the explicit process: Incorporating the intermediate configuration unloaded obeying the elastic constitutive relation from the current configuration; one regards the deformation from the initial to the intermediate configuration to be the plastic deformation and the deformation from the intermediate to the current configuration to be the elastic deformation. Here, note that the elastic deformation is induced from the initial stage in the real deformation behavior as the stress increases but it can be collected in the final stage since it is assumed here to be independent of time. Based on this idea, the multiplicative decomposition of the deformation gradient $\mathbf{F}$ into the elastic part $\mathbf{F}^{\mathrm{e}}$ and the plastic part $\mathbf{F}^{\mathrm{p}}$ was proposed by Kroner (1960) and has been developed by Lee and Liu (1967), Lee (1969), Mandel (1972), Lubarda and Lee (1981), etc. (see Fig. 1) as follows:

$$
\mathbf{F}=\mathbf{F}^{\mathrm{e}} \mathbf{F}^{\mathrm{p}}
$$

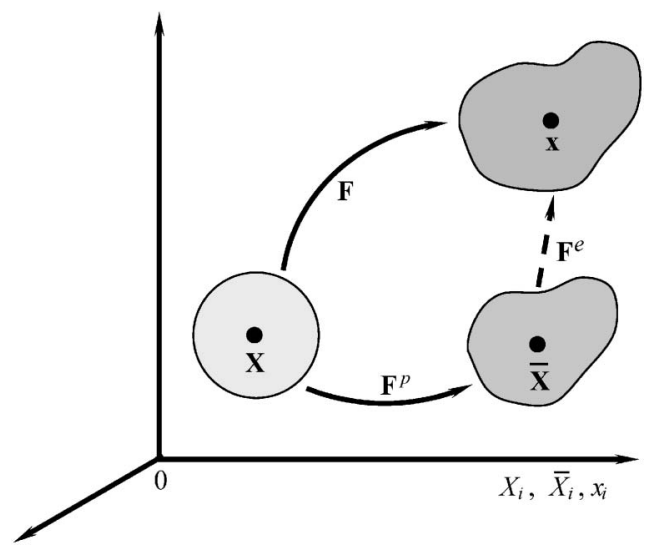

Fig. 1. Multiplicative decomposition of deformation gradient

where

$$
\mathbf{F} \equiv \frac{\partial \mathbf{x}}{\partial \mathbf{X}}, \quad \mathbf{F}^{\mathrm{e}} \equiv \frac{\partial \mathbf{x}}{\partial \mathbf{X}}, \quad \mathbf{F}^{\mathrm{p}} \frac{\partial \overline{\mathbf{X}}}{\partial \mathbf{X}}
$$

$\mathbf{X}$ represents the initial or Lagrangian coordinate of any material point in the continuum under consideration, $\mathbf{x}$ the current or Eulerian coordinate and $\overline{\mathbf{X}}$ the coordinate in the intermediate configuration. Various different plastic deformations are induced and the elastic and plastic deformations are induced in mixed order in real unloading process. However, it should be noted that the real existence of purely elastic unloading process is not required since the intermediate configuration is known by the elastic constitutive relation.

It holds from Eq. (1) that

$$
\operatorname{det} \mathbf{F}=\operatorname{det}\left(\mathbf{F}^{\mathrm{e}} \mathbf{F}^{\mathrm{p}}\right)=\operatorname{det} \mathbf{F}^{\mathrm{e}} \operatorname{det} \mathbf{F}^{\mathrm{p}}
$$

On the other hand, supposing that the line-elements $d \mathbf{X}^{\mathrm{a}}, d \mathbf{X}^{\mathrm{b}}, d \mathbf{X}^{\mathrm{c}}$ in the initial state change to the $d \mathbf{x}^{\mathrm{a}}, d \mathbf{x}^{\mathrm{b}}$, $d \mathbf{x}^{\mathrm{c}}$ in the current state and to $d \overline{\mathbf{X}}^{\mathrm{a}}, d \overline{\mathbf{X}}^{\mathrm{b}}, d \overline{\mathbf{X}}^{\mathrm{c}}$ in the intermediate state, and denoting the initial, the current and the intermediate volume elements as $V, v$ and $\bar{V}$, respectively, it holds that

$$
\begin{aligned}
& d \nu=\left(d \mathbf{x}^{\mathrm{a}} \times d \mathbf{x}^{\mathrm{b}}\right) \cdot d \mathbf{x}^{\mathrm{c}}=\varepsilon_{\mathrm{ijk}} d x_{\mathrm{i}}^{\mathrm{a}} d x_{\mathrm{j}}^{\mathrm{b}} d x_{\mathrm{k}}^{\mathrm{c}}=\operatorname{det} d \mathbf{x} \\
& =\left|\begin{array}{lll}
d x_{1}^{\mathrm{a}} & d x_{1}^{\mathrm{b}} & d x_{1}^{\mathrm{c}} \\
d x_{2}^{\mathrm{a}} & d x_{2}^{\mathrm{b}} & d x_{2}^{\mathrm{c}} \\
d x_{3}^{\mathrm{a}} & d x_{3}^{\mathrm{b}} & d x_{3}^{\mathrm{c}}
\end{array}\right|=\left|\begin{array}{lll}
F_{1 \mathrm{R}} d X_{\mathrm{R}}^{\mathrm{a}} & F_{1 \mathrm{R}} d X_{\mathrm{R}}^{\mathrm{b}} & F_{1 \mathrm{R}} d X_{\mathrm{R}}^{\mathrm{c}} \\
F_{2 \mathrm{R}} d X_{\mathrm{R}}^{\mathrm{a}} & F_{2 \mathrm{R}} d X_{\mathrm{R}}^{\mathrm{b}} & F_{2 \mathrm{R}} d X_{\mathrm{R}}^{\mathrm{c}} \\
F_{3 \mathrm{R}} d X_{\mathrm{R}}^{\mathrm{a}} & F_{3 \mathrm{R}} d X_{\mathrm{R}}^{\mathrm{b}} & F_{3 \mathrm{R}} d X_{\mathrm{R}}^{\mathrm{c}}
\end{array}\right|=\left|\begin{array}{lll}
F_{11} & F_{12} & F_{13} \\
F_{21} & F_{22} & F_{23} \\
F_{31} & F_{32} & F_{33}
\end{array}\right|\left|\begin{array}{lll}
d X_{1}^{\mathrm{a}} & d X_{1}^{\mathrm{b}} & d X_{1}^{\mathrm{c}} \\
d X_{2}^{\mathrm{a}} & d X_{2}^{\mathrm{b}} & d X_{2}^{\mathrm{c}} \\
d X_{3}^{\mathrm{a}} & d X_{3}^{\mathrm{b}} & d X_{3}^{\mathrm{c}}
\end{array}\right|=J d V \\
& d v=\left|\begin{array}{lll}
d x_{1}^{\mathrm{a}} & d x_{1}^{\mathrm{b}} & d x_{1}^{\mathrm{c}} \\
d x_{2}^{\mathrm{a}} & d x_{2}^{\mathrm{b}} & d x_{2}^{\mathrm{c}} \\
d x_{3}^{\mathrm{a}} & d x_{3}^{\mathrm{b}} & d x_{3}^{\mathrm{c}}
\end{array}\right|=\left|\begin{array}{lll}
F_{1 \mathrm{R}}^{\mathrm{e}} d \bar{X}_{\mathrm{R}}^{\mathrm{a}} & F_{1 \mathrm{R}}^{\mathrm{e}} d \bar{X}_{\mathrm{R}}^{\mathrm{b}} & F_{1 \mathrm{R}}^{\mathrm{e}} d \bar{X}_{\mathrm{R}}^{\mathrm{c}} \\
F_{2 \mathrm{R}}^{\mathrm{e}} d \bar{X}_{\mathrm{R}}^{\mathrm{a}} & F_{2 \mathrm{R}}^{\mathrm{e}} d \bar{X}_{\mathrm{R}}^{\mathrm{b}} & F_{2 \mathrm{R}}^{\mathrm{e}} d \bar{X}_{\mathrm{R}}^{\mathrm{c}} \\
F_{3 \mathrm{R}}^{\mathrm{e}} d \bar{X}_{\mathrm{R}}^{\mathrm{a}} & F_{3 \mathrm{R}}^{\mathrm{e}} d \bar{X}_{\mathrm{R}}^{\mathrm{b}} & F_{3 \mathrm{R}}^{\mathrm{e}} d \bar{X}_{\mathrm{R}}^{\mathrm{c}}
\end{array}\right|=\left|\begin{array}{lll}
F_{11}^{\mathrm{e}} & F_{12}^{\mathrm{e}} & F_{13}^{\mathrm{e}} \\
F_{21}^{\mathrm{e}} & F_{22}^{\mathrm{e}} & F_{23}^{\mathrm{e}} \\
F_{31}^{\mathrm{e}} & F_{32}^{\mathrm{e}} & F_{33}^{\mathrm{e}}
\end{array}\right|\left|\begin{array}{lll}
d \bar{X}_{1}^{\mathrm{a}} & d \bar{X}_{1}^{\mathrm{b}} & d \bar{X}_{1}^{\mathrm{c}} \\
d \bar{X}_{2}^{\mathrm{a}} & d \bar{X}_{2}^{\mathrm{b}} & d \bar{X}_{2}^{\mathrm{c}} \\
d \bar{X}_{3}^{\mathrm{a}} & d \bar{X}_{3}^{\mathrm{b}} & d \bar{X}_{3}^{\mathrm{c}}
\end{array}\right|=J^{\mathrm{e}} d \bar{V} \\
& d \bar{V}=\left|\begin{array}{lll}
d \bar{X}_{1}^{\mathrm{a}} & d \bar{X}_{1}^{\mathrm{b}} & d \bar{X}_{1}^{\mathrm{c}} \\
d \bar{X}_{2}^{\mathrm{a}} & d \bar{X}_{2}^{\mathrm{b}} & d \bar{X}_{2}^{\mathrm{c}} \\
d \bar{X}_{3}^{\mathrm{a}} & d \bar{X}_{3}^{\mathrm{b}} & d \bar{X}_{3}^{\mathrm{c}}
\end{array}\right|=\left|\begin{array}{lll}
F_{1 \mathrm{R}}^{\mathrm{p}} d \bar{X}_{\mathrm{R}}^{\mathrm{a}} & F_{1 \mathrm{R}}^{\mathrm{p}} d \bar{X}_{\mathrm{R}}^{\mathrm{b}} & F_{1 \mathrm{R}}^{\mathrm{p}} d \bar{X}_{\mathrm{R}}^{\mathrm{c}} \\
F_{2 \mathrm{R}}^{\mathrm{p}} d \bar{X}_{\mathrm{R}}^{\mathrm{a}} & F_{2 \mathrm{R}}^{\mathrm{p}} d \bar{X}_{\mathrm{R}}^{\mathrm{b}} & F_{2 \mathrm{R}}^{\mathrm{p}} d \bar{X}_{\mathrm{R}}^{\mathrm{c}} \\
F_{3 \mathrm{R}}^{\mathrm{p}} d \bar{X}_{\mathrm{R}}^{\mathrm{a}} & F_{3 \mathrm{R}}^{\mathrm{p}} d \bar{X}_{\mathrm{R}}^{\mathrm{b}} & F_{3 \mathrm{R}}^{\mathrm{p}} d \bar{X}_{\mathrm{R}}^{\mathrm{c}}
\end{array}\right|=\left|\begin{array}{lll}
F_{11}^{\mathrm{p}} & F_{12}^{\mathrm{p}} & F_{13}^{\mathrm{p}} \\
F_{21}^{\mathrm{p}} & F_{22}^{\mathrm{p}} & F_{23}^{\mathrm{p}} \\
F_{31}^{\mathrm{p}} & F_{32}^{\mathrm{p}} & F_{33}^{\mathrm{p}}
\end{array}\right|\left|\begin{array}{lll}
d X_{1}^{\mathrm{a}} & d X_{1}^{\mathrm{b}} & d X_{1}^{\mathrm{c}} \\
d X_{2}^{\mathrm{a}} & d X_{2}^{\mathrm{b}} & d X_{2}^{\mathrm{c}} \\
d X_{3}^{\mathrm{a}} & d X_{3}^{\mathrm{b}} & d X_{3}^{\mathrm{c}}
\end{array}\right|=J^{\mathrm{p}} d V
\end{aligned}
$$


where $\varepsilon_{\mathrm{ijk}}$ stands for the components of the permutation symbol. Eqs. (4)-(6) lead to

$$
J=J^{\mathrm{e}} J^{\mathrm{p}}
$$

where $J$ is the Jacobian between the initial and the current configurations, and $J^{\mathrm{e}}$ and $J^{\mathrm{p}}$ are the elastic and the plastic parts, respectively, of $J$, which are defined as follows:

$$
J \equiv \operatorname{det} \mathbf{F}=\frac{d v}{d V}, \quad J^{\mathrm{e}} \equiv \operatorname{det} \mathbf{F}^{\mathrm{e}}=\frac{d v}{d \bar{V}}, \quad J^{\mathrm{p}} \equiv \operatorname{det} \mathbf{F}^{\mathrm{p}}=\frac{d \bar{V}}{d V}
$$

Eq. (7) leads to the additive decomposition of the logarithmic volumetric strain $\varepsilon_{\mathrm{v}}$ into the elastic logarithmic volumetric strain $\varepsilon_{\mathrm{v}}^{\mathrm{e}}$ and the plastic logarithmic volumetric strain $\varepsilon_{v}^{\mathrm{p}}$, i.e.,

$$
\varepsilon_{\mathrm{v}}=\varepsilon_{\mathrm{v}}^{\mathrm{e}}+\varepsilon_{\mathrm{v}}^{\mathrm{p}}
$$

where

$$
\varepsilon_{\mathrm{v}} \equiv \ln \frac{v}{V}=\ln J, \quad \varepsilon_{\mathrm{v}}^{\mathrm{e}} \equiv \ln \frac{v}{\bar{V}}=\ln J^{\mathrm{e}}, \quad \varepsilon_{\mathrm{v}}^{\mathrm{p}} \equiv \ln \frac{\bar{V}}{V}=\ln J^{\mathrm{p}}
$$

for a homogeneous deformation. It holds from Eqs. (8) and (10) that

$$
\dot{\varepsilon}_{\mathrm{v}}=\frac{\dot{J}}{J}=\frac{\dot{v}}{v}, \quad \dot{\varepsilon}_{\mathrm{v}}^{\mathrm{e}}=\frac{\dot{J}^{\mathrm{e}}}{J^{\mathrm{e}}}=\frac{\dot{v}}{v}-\frac{\dot{\bar{V}}}{\bar{V}}, \quad \dot{\varepsilon}_{\mathrm{v}}^{\mathrm{p}}=\frac{\dot{J}^{\mathrm{p}}}{J^{\mathrm{p}}}=\frac{\dot{\bar{V}}}{\bar{V}}
$$

where $\left(^{\circ}\right)$ denotes the material-time derivative.

Here, note that the intermediate configuration in the general deformation process is not determined uniquely only by the definition as the configuration in the unloaded state, since it depends on a rigid body rotation superimposed in the unloading process to the intermediate configuration. This fact is verified as follows: Letting $\mathbf{Q}$ be an arbitrary orthogonal tensor to represent a rotation, it holds that

$$
\mathbf{F}=\mathbf{F}^{\mathrm{e}} \mathbf{Q}^{\mathrm{T}} \mathbf{Q} \mathbf{F}^{\mathrm{p}}=\hat{\mathbf{F}}^{\mathrm{e}} \hat{\mathbf{F}}^{\mathrm{p}}
$$

where

$$
\hat{\mathbf{F}}^{\mathrm{e}} \equiv \mathbf{F}^{\mathrm{e}} \mathbf{Q}^{\mathrm{T}}, \quad \hat{\mathbf{F}}^{\mathrm{p}} \equiv \mathbf{Q} \mathbf{F}^{\mathrm{p}}
$$

Here, note that $\mathbf{F}^{\mathrm{e}}$ is described by the polar decomposition into the symmetric tensor $\mathbf{V}^{\mathrm{e}}$ and the orthogonal tensor $\mathbf{R}^{\mathrm{e}}$ as follows:

$$
\mathbf{F}^{\mathrm{e}}=\mathbf{V}^{\mathrm{e}} \mathbf{R}^{\mathrm{e}}
$$

where

$$
\begin{aligned}
& \mathbf{V}^{\mathrm{e}}=\left(\mathbf{F}^{\mathrm{e}} \mathbf{F}^{\mathrm{eT}}\right)^{1 / 2}\left(\mathbf{V}^{\mathrm{e}}=\mathbf{V}^{\mathrm{eT}}\right), \\
& \mathbf{R}^{\mathrm{e}}=\left(\mathbf{F}^{\mathrm{e}} \mathbf{F}^{\mathrm{T}}\right)^{-1 / 2}\left(\mathbf{R}^{\mathrm{e}}=\mathbf{R}^{\mathbf{e}^{-\mathrm{T}}}\right)
\end{aligned}
$$

In order to exclude this nonuniqueness of $\mathbf{F}^{\mathrm{e}}$ in Eq. (14) depending on the arbitrariness in selection of $\mathbf{R}^{\mathrm{e}}$, it is assumed that the imaginary unloading process to the intermediate configuration is the pure deformation so that

$$
\mathbf{F}^{\mathrm{e}}=\mathbf{V}^{\mathrm{e}}, \quad \mathbf{R}^{\mathrm{e}}=\mathbf{I}
$$

where $\mathbf{I}$ is the identity tensor having the components of Kronecker's delta $\delta_{\mathrm{ij}}$, i.e., $\delta_{\mathrm{ij}}=1$ for $i=j$ and $\delta_{\mathrm{ij}}=0$ for $i \neq j$. Then, $\mathbf{F}^{\mathrm{e}}$ becomes the symmetric tensor, i.e., $\mathbf{F}^{\mathrm{e}}=$ $\mathbf{F}^{\mathrm{eT}}$.

The velocity gradient $\mathbf{L}$ is defined by

$$
\mathbf{L} \equiv \frac{\partial \dot{\mathbf{x}}}{\partial \mathbf{x}}=\dot{\mathbf{F}} \mathbf{F}^{-1}
$$

The substitutions of Eqs. (1) and (16) into Eq. (17) leads to

$$
\mathbf{L}=\dot{\mathbf{V}}^{\mathrm{e}} \mathbf{V}^{\mathrm{e}-1}+\mathbf{V}^{\mathrm{e}} \dot{\mathbf{F}}^{\mathrm{p}} \mathbf{F}^{\mathrm{p}-1} \mathbf{V}^{\mathrm{e}-1}
$$

and thus

$$
\mathbf{L}=\mathbf{L}^{\mathrm{e}}+\tilde{\mathbf{L}}^{\mathrm{p}}
$$

where

$$
\left.\begin{array}{rl}
\mathbf{L}^{\mathrm{e}} & \equiv \dot{\mathbf{V}}^{\mathrm{e}} \mathbf{V}^{\mathrm{e}-1}=\left(\frac{\partial \mathbf{x}}{\partial \overline{\mathbf{X}}}\right)^{\bullet} \frac{\partial \overline{\mathbf{X}}}{\partial \mathbf{x}} \\
\mathbf{L}^{\mathrm{p}} & \equiv \dot{\mathbf{F}}^{\mathrm{p}} \mathbf{F}^{\mathrm{p}-1}=\frac{\partial \dot{\mathbf{X}}}{\partial \mathbf{X}} \frac{\partial \mathbf{X}}{\partial \overline{\mathbf{X}}}=\frac{\partial \dot{\mathbf{X}}}{\partial \overline{\mathbf{X}}} \\
\tilde{\mathbf{L}}^{\mathrm{p}} & \equiv \mathbf{V}^{\mathrm{e}} \mathbf{L}^{\mathrm{p}} \mathbf{V}^{\mathrm{e}-1}=\frac{\partial \mathbf{x}}{\partial \overline{\mathbf{X}}} \frac{\partial \dot{\mathbf{X}}}{\partial \overline{\mathbf{X}}} \frac{\partial \overline{\mathbf{X}}}{\partial \mathbf{x}}
\end{array}\right\}
$$

Further, $\mathbf{L}^{\mathrm{e}}, \mathbf{L}^{\mathrm{p}}, \tilde{\mathbf{L}}^{\mathrm{p}}$ can be additively decomposed into the symmetric and the skew-symmetric parts as follows:

$$
\left.\begin{array}{rl}
\mathbf{L}^{\mathrm{e}} & =\mathbf{D}^{\mathrm{e}}+\mathbf{W}^{\mathrm{e}} \\
\mathbf{L}^{\mathrm{p}} & =\mathbf{D}^{\mathrm{p}}+\mathbf{W}^{\mathrm{p}} \\
\tilde{\mathbf{L}}^{\mathrm{p}} & =\tilde{\mathbf{D}}^{\mathrm{p}}+\tilde{\mathbf{W}}^{\mathrm{p}}
\end{array}\right\}
$$

where

$$
\begin{aligned}
& \mathbf{D}^{\mathrm{e}}=\left(\mathbf{L}^{\mathrm{e}}\right)_{\mathrm{s}}=\left(\dot{\mathbf{V}}^{\mathrm{e}} \mathbf{V}^{\mathrm{e}-1}\right)_{\mathrm{s}}, \quad \mathbf{W}^{\mathrm{e}}=\left(\mathbf{L}^{\mathrm{e}}\right)_{\mathrm{a}}=\left(\dot{\mathbf{V}}^{\mathrm{e}} \mathbf{V}^{\mathrm{e}-1}\right)_{\mathrm{a}} \\
& \mathbf{D}^{\mathrm{p}}=\left(\mathbf{L}^{\mathrm{p}}\right)_{\mathrm{s}}=\left(\dot{\mathbf{F}}^{\mathrm{p}} \mathbf{F}^{\mathrm{p}-1}\right)_{\mathrm{s}}, \quad \mathbf{W}^{\mathrm{p}}=\left(\mathbf{L}^{\mathrm{p}}\right)_{\mathrm{a}}=\left(\dot{\mathbf{F}}^{\mathrm{p}} \mathbf{F}^{\mathrm{p}-1}\right)_{\mathrm{a}} \\
& \tilde{\mathbf{D}}^{\mathrm{p}}=\left(\tilde{\mathbf{L}}^{\mathrm{p}}\right)_{\mathrm{s}}=\left(\mathbf{V}^{\mathrm{e}} \dot{\mathbf{F}}^{\mathrm{p}} \mathbf{F}^{\mathrm{p}-1} \mathbf{V}^{\mathrm{e}-1}\right)_{\mathrm{s}}, \\
& \tilde{\mathbf{W}}^{\mathrm{p}}=\left(\tilde{\mathbf{L}}^{\mathrm{p}}\right)_{\mathrm{a}}=\left(\mathbf{V}^{\mathrm{e}} \dot{\mathbf{F}}^{\mathrm{p}} \mathbf{F}^{\mathrm{p}-1} \mathbf{V}^{\mathrm{e}-1}\right)_{\mathrm{a}}
\end{aligned}
$$

where ()$_{s}$ and ()$_{a}$ denote the symmetric and the skewsymmetric parts, respectively.

The strain rate $\mathbf{D}$ and the spin $\mathbf{W}$ are additively decomposed from Eq. (19) as follows:

$$
\begin{aligned}
\mathbf{D} \equiv & \frac{1}{2}\left(\mathbf{L}+\mathbf{L}^{\mathrm{T}}\right)=\mathbf{D}^{\mathrm{e}}+\tilde{\mathbf{D}}^{\mathrm{p}}=\mathbf{D}^{\mathrm{e}}+\left(\mathbf{V}^{\mathrm{e}} \mathbf{D}^{\mathrm{p}} \mathbf{V}^{\mathrm{e}-1}\right)_{\mathrm{s}} \\
& +\left(\mathbf{V}^{\mathrm{e}} \mathbf{W}^{\mathrm{p}} \mathbf{V}^{\mathrm{e}-1}\right)_{\mathrm{s}} \\
\mathbf{W} \equiv & \frac{1}{2}\left(\mathbf{L}-\mathbf{L}^{\mathrm{T}}\right)=\mathbf{W}^{\mathrm{e}}+\tilde{\mathbf{W}}^{\mathrm{p}}=\mathbf{W}^{\mathrm{e}}+\left(\mathbf{V}^{\mathrm{e}} \mathbf{D}^{\mathrm{p}} \mathbf{V}^{\mathrm{e}-1}\right)_{\mathrm{a}} \\
& +\left(\mathbf{V}^{\mathrm{e}} \mathbf{W}^{\mathrm{p}} \mathbf{V}^{\mathrm{e}-1}\right)_{\mathrm{a}}
\end{aligned}
$$

Further, the following equations hold for the volumetric strain rate from Eqs. (11), (17) and (22)-(25).

$$
\begin{aligned}
& D_{\mathrm{v}} \equiv \operatorname{tr} \mathbf{D}=\dot{\varepsilon}_{\mathrm{v}}=\operatorname{tr} \frac{\partial \dot{\mathbf{x}}}{\partial \mathbf{x}}=\frac{\partial \dot{x}_{\mathrm{i}}}{\partial x_{\mathrm{i}}}=\frac{\left(\partial x_{1} \partial x_{2} \partial x_{3}\right)^{\bullet}}{\partial x_{1} \partial x_{2} \partial x_{3}}=\frac{\dot{v}}{v} \\
& D_{\mathrm{v}}^{\mathrm{p}} \equiv \operatorname{tr} \mathbf{D}^{\mathrm{p}}=\dot{\varepsilon}_{\mathrm{v}}^{\mathrm{e}}=\operatorname{tr} \frac{\partial \dot{\mathbf{X}}}{\partial \overline{\mathbf{X}}}=\frac{\partial \dot{\bar{X}}}{\partial \bar{X}_{\mathrm{i}}}=\frac{\left(\partial \bar{X}_{1} \partial \bar{X}_{2} \partial \bar{X}_{3}\right)^{\bullet}}{\partial \bar{X}_{1} \partial \bar{X}_{2} \partial \bar{X}_{3}}=\frac{\dot{\bar{V}}}{\bar{V}} \\
& {\tilde{D_{\mathrm{v}}^{\mathrm{p}}}}^{\mathrm{p}} \operatorname{tr} \tilde{\mathbf{D}}^{\mathrm{p}}=\operatorname{tr}\left(\frac{\partial \mathbf{x}}{\partial \overline{\mathbf{X}}} \frac{\partial \dot{\mathbf{X}}}{\partial \overline{\mathbf{X}}} \frac{\partial \overline{\mathbf{X}}}{\partial \mathbf{x}}\right)=\operatorname{tr} \frac{\partial \dot{\mathbf{X}}}{\partial \overline{\mathbf{X}}}=\frac{\dot{\bar{V}}}{\bar{V}}=D_{\mathrm{v}}^{\mathrm{p}} \\
& D_{\mathrm{v}}^{\mathrm{e}} \equiv \operatorname{tr} \mathbf{D}^{\mathrm{e}}=\dot{\varepsilon}_{\mathrm{v}}^{\mathrm{p}}=\operatorname{tr} \mathbf{D}-\operatorname{tr} \tilde{\mathbf{D}}^{\mathrm{p}}=\frac{\dot{v}}{v}-\frac{\dot{\bar{V}}}{\bar{V}}
\end{aligned}
$$

from which the additive decomposition holds exactly for the volumetric strain rate, i.e., 


$$
D_{\mathrm{v}}=D_{\mathrm{v}}^{\mathrm{e}}+D_{\mathrm{v}}^{\mathrm{p}}
$$

On the other hand, the additive decomposition of the strain rate tensor does not hold because of $\mathbf{D}^{p} \neq \tilde{\mathbf{D}}^{\mathrm{p}}$ except for the particular case without the elastic deformation, i.e., $\mathbf{V}^{\mathrm{e}}=\mathbf{I}$. However, the elastic deformation is quite small compared with the plastic deformation and one can put $\mathbf{V}^{\mathrm{e}} \cong \mathbf{I}$ in some solid materials including metals. In such case, the following equations hold approximately.

$$
\left.\begin{array}{l}
\mathbf{D}=\mathbf{D}^{\mathrm{e}}+\mathbf{D}^{\mathrm{p}}\left(\mathbf{D}^{\mathrm{e}}=\dot{\mathbf{V}}^{\mathrm{e}}\right) \\
\mathbf{W}=\mathbf{W}^{\mathrm{p}}
\end{array}\right\}
$$

\section{ISOTROPIC CONSOLIDATION OF SOILS}

The linear relation of void ratio $v s$. logarithm of pressure has been most widely adopted to constitutive equations of soils. On the other hand, the linear relation of both logarithms of volume and pressure was proposed by Hashiguchi (1974) and adopted for the formulation of subloading surface model of soils (Hashiguchi and Ueno, 1977; Hashiguchi and Chen, 1998; Hashiguchi et al., 2002). In this section they are examined on the physical and mathematical pertinence, especially on the fulfillment of the multiplicative decomposition of deformation gradient.

\section{Linear Relation of Both Logarithms of Volume and Pres- sure}

The $\ln v-\ln p$ linear relation, i.e., the linear relationship between both logarithms of volume $v$ and pressure $p$ has been proposed by the author (Hashiguchi, 1974, 1985, 1995; Hashiguchi and Ueno, 1977; Hashiguchi et al., 2002) in which the normal-consolidation and the swelling lines are given by

$$
\begin{aligned}
& \text { Normal-consolidated line: } \ln \frac{v_{\mathrm{y}}}{v_{\mathrm{y} 0}}=-\rho \ln \frac{p_{\mathrm{y}}}{p_{\mathrm{y} 0}} \\
& \text { Swelling line: } \ln \frac{v}{\bar{V}}=-\gamma \ln \frac{p}{p_{0}}
\end{aligned}
$$

where the material constants $\rho$ and $\gamma$ are the slopes of normal-consolidation and swelling lines, respectively, in the $(\ln v, \ln p)$ plane. $p_{0}$ is the initial pressure. $p_{\mathrm{y} 0}, v_{\mathrm{y} 0}$ and $p_{\mathrm{y}}, v_{\mathrm{y}}$ are the pressures, the volumes in the initial yield state and in the current yield state, respectively.

It should be emphasized that $v$ is not the specific volume $1+e$ ( $e$ : specific volume) which cannot be related exactly to the volumetric strain but is the volume itself which is related directly and strictly to it. The pertinence of the isotropic consolidation characteristics in Eq. (29) was discussed later by Butterfield (1979), whilst the volume itself was not used but the specific volume was used, and thus the exact formulation was not given in the discussions.

Translating the range of pressure to the negative direction of the $p$-axis by the quantity $p_{\mathrm{e}}(\geq 0)$ (Fig. 2), Eq. (29) leads to

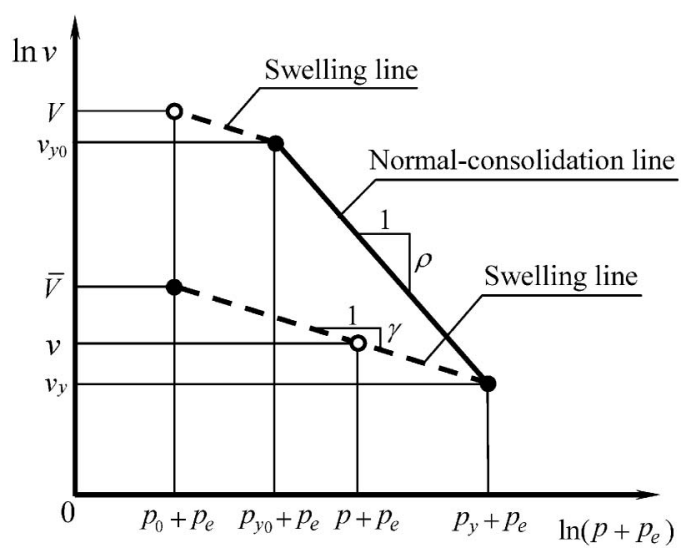

Fig. 2. In $v-\ln p$ linear relation of isotropic consolidation of soils

Normal-consolidated line: $\ln \frac{v_{\mathrm{y}}}{v_{\mathrm{y} 0}}=-\rho \ln \frac{p_{\mathrm{y}}+p_{\mathrm{e}}}{p_{\mathrm{y} 0}+p_{\mathrm{e}}}$

Swelling line: $\ln \frac{v}{\bar{V}}=-\gamma \ln \frac{p+p_{\mathrm{e}}}{\mathrm{p}_{0}+p_{\mathrm{e}}}$

where $p_{\mathrm{e}}$ is regarded to be the material constant hereinafter, whilst it holds that $v \rightarrow \infty$ for $p \rightarrow-p_{\mathrm{e}}$.

It holds from Eqs. (10) and (30) that

$$
\begin{aligned}
\varepsilon_{\mathrm{v}}= & \varepsilon_{\mathrm{v}}^{\mathrm{e}}+\varepsilon_{\mathrm{v}}^{\mathrm{p}}=\ln \frac{v}{\bar{V}}+\ln \frac{\bar{V}}{V}=\ln \frac{v}{\bar{V}}+\left(\ln \frac{v_{\mathrm{y} 0}}{V}+\ln \frac{v_{\mathrm{y}}}{v_{\mathrm{y} 0}}+\ln \frac{\bar{V}}{v_{\mathrm{y}}}\right) \\
= & -\gamma \ln \frac{p+p_{\mathrm{e}}}{p_{0}+p_{\mathrm{e}}}+\left(-\gamma \ln \frac{p_{\mathrm{y} 0}+p_{\mathrm{e}}}{p_{0}+p_{\mathrm{e}}}-\rho \ln \frac{p_{\mathrm{y}}+p_{\mathrm{e}}}{p_{\mathrm{y} 0}+p_{\mathrm{e}}}\right. \\
& \left.-\gamma \ln \frac{p_{0}+p_{\mathrm{e}}}{p_{\mathrm{y}}+p_{\mathrm{e}}}\right)=-\gamma \ln \frac{p+p_{\mathrm{e}}}{p_{0}+p_{\mathrm{e}}}-(\rho-\gamma) \ln \frac{p_{\mathrm{y}}+p_{\mathrm{e}}}{p_{\mathrm{y} 0}+p_{\mathrm{e}}}
\end{aligned}
$$

i.e.,

$$
\begin{aligned}
\varepsilon_{\mathrm{v}} & =-\gamma \ln \frac{p+p_{\mathrm{e}}}{p_{0}+p_{\mathrm{e}}}-(\rho-\gamma) \ln \frac{p_{\mathrm{y}}+p_{\mathrm{e}}}{p_{\mathrm{y} 0}+p_{\mathrm{e}}}, \\
\varepsilon_{\mathrm{v}}^{\mathrm{e}} & =-\gamma \ln \frac{p+p_{\mathrm{e}}}{p_{0}+p_{\mathrm{e}}} \\
\varepsilon_{\mathrm{v}}^{\mathrm{p}} & =-(\rho-\gamma) \ln \frac{p_{\mathrm{y}}+p_{\mathrm{e}}}{p_{\mathrm{y} 0}+p_{\mathrm{e}}}
\end{aligned}
$$

The elastic and the plastic volumetric strain rates are given from Eq. (32) as follows:

$$
\begin{aligned}
& D_{\mathrm{v}}=-\gamma \frac{\dot{p}}{p_{0}+p_{\mathrm{e}}}-(\rho-\gamma) \frac{\dot{p}_{\mathrm{y}}}{p_{\mathrm{y}}+p_{\mathrm{e}}}, \\
& D_{\mathrm{v}}^{\mathrm{e}}=-\gamma \frac{\dot{p}}{p_{0}+p_{\mathrm{e}}}, \\
& D_{\mathrm{v}}^{\mathrm{p}}=-(\rho-\gamma) \frac{\dot{p}_{\mathrm{y}}}{p_{\mathrm{y}}+p_{\mathrm{e}}}
\end{aligned}
$$

which show the volumetric strain rate and the volumetric elastic and the plastic strain rates derived exactly from the $\ln v-\ln p$ linear relation based on the multiplicative decomposition.

The elastic deformation characteristic is influenced by the plastic deformation in general but $p_{\mathrm{y}}$ is not incorporated in Eqs. (32) and (33) for the elastic volumetric strain 
and its rate in order to avoid the elastic-plastic coupling causing the complexity of formulation, whilst the coupling in soils is not so strong as in brittle geomaterials, e.g., rocks and concretes.

For the formulation of constitutive equation of soils Borja and Tamagnini (1998) used the equation which has the identical form as Eq. (29) with the replacement of $p$ to the pressure of Kirchoff stress $\tau=J \sigma$ and described that Eq. (33) without $p_{\mathrm{e}}$ is obtained from that equation. However, it cannot be derived exactly since the relation $\dot{J}_{\mathrm{y}}$ $/ J_{\mathrm{y}}=\dot{v}_{\mathrm{y}} / v_{\mathrm{y}}=\dot{J}^{\mathrm{p}} / J^{\mathrm{p}}$ does not hold in general but holds only in the normal-consolidation process although this relation was used in the derivation. In addition, they have not used the volume itself but adopted the specific volume as has been done by Butterfield (1979).

Linear Relation of Void Ratio vs. Logarithm of Pressure

The $e-\ln p$ linear relation for the isotropic consolidation has been widely adopted for constitutive equation of soils including Cam-clay models (Roscoe and Burland, 1968; Schofield and Wroth, 1968) in which the normalconsolidation and the swelling lines are given by

$$
\left.\begin{array}{l}
\text { Normal-consolidated line: } e_{\mathrm{y}}-e_{\mathrm{yo}}=-\lambda \ln \frac{p_{\mathrm{y}}}{p_{\mathrm{y} 0}} \\
\text { Swelling line: } e-\bar{e}=-\kappa \ln \frac{p}{p_{0}}
\end{array}\right\}
$$

where the material constants $\lambda$ and $\kappa$ are the slopes of normal-consolidation and swelling lines, respectively, in $(e, \ln p)$ space (Fig. 3), where $p_{0}, e_{0} ; p_{\mathrm{y} 0}, e_{\mathrm{y} 0}$ and $p_{\mathrm{y}}, e_{\mathrm{y}}$ are the pressure, the void ratio in the initial, the initial yield and the current yield states, respectively. $\bar{e}$ is the void ratio in the unloaded state to the initial pressure $p_{0}$. This relation has the following physical irrationalities.

1. The change of void ratio induced during a same range of pressure along the swelling line is independent of a pre-consolidation pressure $p_{\mathrm{y}}$ with which the plastic decrease of void ratio proceeds.

2. The void ratio becomes negative if the pressure becomes large as $p_{\mathrm{y}}>p_{\mathrm{y} 0} \exp \left(e_{\mathrm{y} 0} / \lambda\right)$ or $p>p_{0} \exp$ $(\bar{e} / \lambda)$.

3. The relation is not given by the volume but by the void

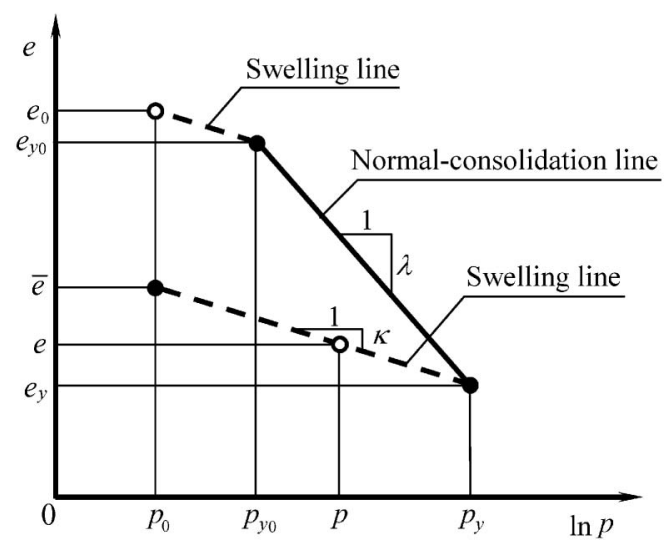

Fig. 3. $e$-In $p$ linear relation of isotropic consolidation of soils ratio and thus it is quite difficult to derive the exact relation of pressure $v s$. volumetric strain even if the compressibility property of soil particles is given.

Further, unfortunately the relation of pressure vs. logarithmic volumetric strain becomes quite complex. Therefore, the following nominal volumetric strain has been adopted in constitutive equation.

$$
\begin{aligned}
& \bar{\varepsilon}_{\mathrm{v}}=\frac{v-V}{V}, \\
& \bar{\varepsilon}_{\mathrm{v}}^{\mathrm{e}}=\frac{v-\bar{V}}{V}, \\
& \bar{\varepsilon}_{\mathrm{v}}^{\mathrm{p}}=\frac{\bar{V}-V}{V}
\end{aligned}
$$

Here, it should be noted that the nominal strain is irrelevant to the strain rate $\mathbf{D}$ and thus $\bar{\varepsilon}_{\mathrm{v}} \neq \varepsilon_{\mathrm{v}}, \bar{\varepsilon}_{\mathrm{v}}^{\mathrm{e}} \neq \varepsilon_{\mathrm{v}}^{\mathrm{e}}, \bar{\varepsilon}_{\mathrm{v}}^{\mathrm{p}} \neq$ $\varepsilon_{\mathrm{v}}^{\mathrm{p}}$. The following approximation is used by substituting into Eq. (35) the void ratio instead of the volume in order to avoid the above-mentioned deficiency 3 .

$$
\left.\begin{array}{rl}
\bar{\varepsilon}_{\mathrm{v}} & \cong \frac{e-e_{0}}{1+e_{0}}, \\
\bar{\varepsilon}_{\mathrm{v}}^{\mathrm{e}} \cong \frac{e-\bar{e}_{0}}{1+e_{0}}, \\
\bar{\varepsilon}_{\mathrm{v}}^{\mathrm{p}} \cong \frac{\bar{e}-e_{0}}{1+e_{0}}=\frac{\left(e_{\mathrm{y} 0}-e_{0}\right)+\left(\bar{e}_{\mathrm{y}}-e_{\mathrm{y} 0}\right)+\left(\bar{e}-e_{\mathrm{y}}\right)}{1+e_{0}}
\end{array}\right\}
$$

Substituting Eq. (34) into Eq. (36), one has

$$
\begin{aligned}
& \bar{\varepsilon}_{\mathrm{v}} \cong-\frac{\kappa}{1+e_{0}} \ln \frac{p}{p_{0}}-\frac{\lambda-\kappa}{1+e_{0}} \ln \frac{p_{\mathrm{y}}}{p_{\mathrm{y} 0}}, \\
& \bar{\varepsilon}_{\mathrm{v}}^{\mathrm{e}} \cong-\frac{\kappa}{1+e_{0}} \ln \frac{p}{p_{0}}, \\
& \bar{\varepsilon}_{\mathrm{v}}^{\mathrm{p}} \cong-\frac{\kappa}{1+e_{0}} \ln \frac{p_{\mathrm{y} 0}}{p_{0}}-\frac{\lambda}{1+e_{0}} \ln \frac{p_{\mathrm{y}}}{p_{\mathrm{y} 0}}-\frac{\kappa}{1+e_{0}} \ln \frac{p_{0}}{p_{\mathrm{y}}}= \\
&-\frac{\lambda-\kappa}{1+e_{0}} \ln \frac{p_{\mathrm{y}}}{p_{\mathrm{y} 0}}
\end{aligned}
$$

from which the nominal volumetric strain rate is given by

$$
\begin{aligned}
& \dot{\bar{\varepsilon}}_{\mathrm{v}}\left(\cong D_{\mathrm{v}}\right) \cong-\frac{\kappa}{1+e_{0}} \frac{\dot{p}}{p}-\frac{\lambda-\kappa}{1+e_{0}} \frac{\dot{p}_{\mathrm{y}}}{p_{\mathrm{y}}}, \\
& \dot{\bar{\varepsilon}}_{\mathrm{v}}^{\mathrm{e}}\left(\cong D_{\mathrm{v}}^{\mathrm{e}}\right) \cong-\frac{\kappa}{1+e_{0}} \frac{\dot{p}}{p}, \\
& \dot{\bar{\varepsilon}}_{\mathrm{v}}^{\mathrm{p}}\left(\cong D_{\mathrm{v}}^{\mathrm{p}}\right) \cong-\frac{\lambda-\kappa}{1+e_{0}} \frac{\dot{p}_{\mathrm{y}}}{p_{\mathrm{y}}}
\end{aligned}
$$

Eq. (38) is adopted most widely for elastoplastic constitutive equations of soils. However, it has the following deficiencies.

4. It is derived merely approximately from the $e-\ln p$ linear relation.

5. It cannot be adopted to describe the finite deformation since it is derived based on the definition of nominal strain which is holds only for the description of an infinitesimal deformation.

6. The tangent elastic bulk modulus $K\left(\equiv \dot{p} / \dot{\bar{\varepsilon}}_{\mathrm{v}}^{\mathrm{e}}\right)$ is given by $K=\left(1+e_{0}\right) p / \kappa$ from Eq. (38). It has the crucial 
physical impertinence: "Larger the void ratio, larger is the elastic bulk modulus, i.e., Looser the soil, harder is the change of void ratio to be induced". In addition, the tangent elastic bulk modulus $K$ depends on the initial void ratio $e_{0}$ and thus is not a material constant, lacking the generality.

Eventually, it is concluded that the $e-\ln p$ linear relation is not adequate physically and mathematically for the formulation of constitutive equation for a finite deformation of soils. Needless to say, it results in the physical impertinence that the void ratio becomes infinite for the null stress as far as the translation given for Eq. (30) is not furnished.

These various deficiencies in the $e-\ln p$ linear relation are remedied in the $\ln v-\ln p$ linear relation.

\section{CONCLUDING REMARKS}

The isotropic consolidation characteristics influence heavily the deformation behavior of soils. The equations for isotropic consolidation of soils are examined as to the exact fulfillment of the multiplicative decomposition of deformation gradient in detail. Then, it is verified that the pertinent relation of logarithmic volume strain $v s$. logarithm of pressure in the simple form is derived from the linear relation of both logarithms of volume and pressure based on the multiplicative decomposition of deformation gradient. On the other hand, a pertinent relation of volumetric strain $v s$. pressure is not derived from the linear relation of void ratio $v s$. logarithm of pressure although it has been adopted most widely for constitutive equations of soils.

As far as the linear relation of void ratio vs. logarithm of pressure is used, constitutive equations applicable to the description of the finite deformation of soils would not be obtained forever, although it is widely used even at present while it passed already more than thirty years after the proposition of the linear relation of both logarithms of volume and pressure. It should be recommended strongly to adopt the linear relation of both logarithms of volume and pressure for the steady development of geomechanics.

\section{REFERENCES}

1) Borja, R. I. and Tamagnini, C. (1998): Cam-clay plasticity, Part III: Extension of the infinitesimal model to include finite strains, Comput. Methods Appl. Mech. Eng., 155, 73-95.

2) Butterfield, R. (1979): A natural compression law for soils, Geotechnique, 29, 469-480.

3) Dafalias, Y. F. (1983): Corotational rates for kinematic hardening at large plastic deformations, J. Appl. Mech, ASME, 50, 651-565.

4) Dafalias, Y. F. (1985): The plastic spin, J. Appl., ASME, 52, 865-871.

5) Drucker, D. C. and Prager, W. (1952): Soil mechanics and plastic analysis or limit design, Quart. Appl. Math., 10, 157-165.

6) Hashiguchi, K. (1974): Isotropic hardening theory of granular media, Proc. Japan Soc. Civil Eng., (227), 45-60.

7) Hashiguchi, K. and Ueno, M. (1977): Elastoplastic constitutive laws of granular materials, Constitutive Equations of Soils, Proc. 9th ICSMFE, Spec. Session 9, Tokyo, JSSMFE, Tokyo, 73-82.

8) Hashiguchi, K. (1985): Macrometric approaches-static-intrinsically time-independent, Constitutive Laws of Soils, Proc. 11th ICSMFE, Disc. Sess. IA, San Francisco, 25-65.

9) Hashiguchi, K. (1995): On the linear relations of $V$ - $\ln p$ and $\ln v$ $\ln p$ for isotropic consolidation of soils, Int. J. Numer. Anal. Meth. Geomech., 19, 367-376.

10) Hashiguchi, K. and Chen, Z.-P. (1998): Elastoplastic constitutive equations of soils with the subloading surface and the rotational hardening, Int. J. Numer. Anal. Meth. Geomech., 22, 197-227.

11) Hashiguchi, K., Saitoh, K., Okayasu, T. and Tsutsumi, S. (2002): Evaluation of typical conventional and unconventional plasticity models for prediction of softening behavior of soils, Geotechnique, 52, 561-573.

12) Hashiguchi, K. (2007): General corotational rate tensor and replacement of material-time derivative to corotational derivative of yield function, Comput. Model. Eng. Sci., 17(1), 55-62.

13) Lee, E. H. and Liu, D. T. (1967): Finite-strain elastic-plastic theory with application to plane-wave analysis, J. Appl. Phys., 38, 19-27.

14) Lee, E. H. (1969): Elastic-plastic deformation at finite strains, $J$. Appl. Mech., ASME, 36, 1-6.

15) Kroner, E. (1960): Allgemeine Kontinuumstheoreie der Versetzungen und Eigenspannnungen, Arch. Ration. Mech. Anal., 4, 273-334.

16) Mandel, G. (1972): Plastidite classique et viscoplasticite, CISM Course, Springer-Verlsg.

17) Roscoe, K. H. and Burland, J. B. (1968): On the generalized stressstrain behavior of 'wet' clay, Engineering Plasticity (eds. by Heyman, J. and Leckie, F. A.), Cambridge Univ. Press, 535-608.

18) Schofield, A. N. and Wroth, C. P. (1968): Critical State Soil Mechanics, McGraw-Hill, London.

19) Simo, J. C. and Meschke, G. (1993): A new class of algorithms for classical plasticity extended to finite strains, Application to geomaterials, Comp. Mech., 11, 253-278. 